\title{
Mathematical Methods Applied to the Celestial Mechanics of Artificial Satellites 2014
}

\author{
Antonio F. Bertachini A. Prado, ${ }^{1}$ Josep J. Masdemont, ${ }^{2}$ Maria Cecilia Zanardi, ${ }^{3}$ \\ Silvia Maria Giuliatti Winter, ${ }^{3}$ Tadashi Yokoyama, ${ }^{4}$ and Vivian Martins Gomes $^{3}$ \\ ${ }^{1}$ INPE-DMC, Avenida dos Astronautas 1758, 12227-010 São José dos Campos, SP, Brazil \\ ${ }^{2}$ IEEC and Departament de Matemàtica Aplicada I, Universitat Politècnica de Catalunya, Diagonal 647, 08028 Barcelona, Spain \\ ${ }^{3}$ Universidade Estadual Paulista-(UNESP), Campus de Guaratinguetá, 12516-410 Guaratinguetá, SP, Brazil \\ ${ }^{4}$ Universidade Estadual Paulista-(UNESP), Campus de Rio Claro, Caixa Postal 178, 13500-970 Rio Claro, SP, Brazil
}

Correspondence should be addressed to Antonio F. Bertachini A. Prado; bertachiniprado@bol.com.br

Received 2 October 2014; Accepted 2 October 2014

Copyright (C) 2015 Antonio F. Bertachini A. Prado et al. This is an open access article distributed under the Creative Commons Attribution License, which permits unrestricted use, distribution, and reproduction in any medium, provided the original work is properly cited.

\begin{abstract}
Astrodynamics is a field of study that concentrates itself on the motion of spacecraft governed by natural and man-made forces. The most common of the natural forces are the gravity of the celestial bodies, the radiation pressure that comes from the stars, the drag and lift originated by the atmosphere of the planets, and so forth. The man-made forces are concentrated in propulsion forces, which are the result of an engine included in the spacecraft with the goal of modifying its orbit or orientation in space. Most of those studies come from the astronomy field, in particular the Celestial Mechanics, which has been existing for a long time. It started with the observation of the sky, when the first astronomers tried to explain the motion of some particular stars against the large number of "fixed" stars that composed the background. They were called "planets" by the Greeks, which means "errants" due to their motion against the other "fixed stars." Several of the most famous names in the history of science worked on that field, like Nicolaus Copernicus, Galileo Galilei, Johannes Kepler, Isaac Newton, Albert Einstein, just to point out the more recent names. They all helped to find the physical laws that govern the motions of celestial bodies.
\end{abstract}

The astrodynamics has a very short history when compared to astronomy and other related sciences, but it helped to change the lives of people on Earth. The so-called "space age" had its first important mark when the first artificial satellite orbited the Earth and was able to send the first man-made signs from space. It was built and launched by the former Soviet Union, in the year 1957. It was named Sputnik. Many other important steps were taken very fast, in particular due to the strong battle between the United States of America and the Soviet Union, that was known as the "Cold War." The first man launched to space was also achieved by the Soviet Union, in 1961, with the historical flight of Yuri Gagarin. The United States of America also made its steps in space, with the first American satellite (Explorer I) launched in 1958 and the first American to travel in space (Alan Sheppard) in 1961. The competition then moved to reach the Moon and, after several automated spacecraft travelled to our natural satellite, the Americans Neil Armstrong (the first astronaut) and Edwin Aldrin (the second astronaut) set their feet on the Moon in 1969. Afterwards, a very intensive effort was then made to visit all the planets of the Solar System, as well as many of their moons.

Besides the important steps made in science, daily life on Earth was also improved by space activities. The explosion of fast communications all over the world by telephone, television, internet, and so forth, is largely based on space. More recently, constellations of satellites, like GLONASS, Beidou, and GPS, started their service of positioning and helped people to locate places using small and cheap receivers or even their mobile phones. Important resources on Earth are also discovered from space. 
The weather forecast would be impossible without the use of satellites, not only helping people to plan their day, but also giving important contributions to increase efficiency in agricultural activities and in the prediction of natural disaster.

The present special issue is the fourth special issue covering activities related to space research, which shows the importance of this topic. It includes subjects related to several fields on astrodynamics, including attitude and orbit propagation, orbit design, and control of spacecraft.

P. C. P. M. Pardal et al. studied frozen orbits by deriving analytical expressions for the variation of the eccentricity and argument of perigee, which includes the secular and long period components. The effects of the geopotential and the atmospheric drag were taken into account in this system of nonlinear differential equations. The analysis was carried out by studying the orbital evolution of the CBERS (China Brazil Earth Resources Satellite) satellites family and similar satellites: SPOT, Landsat, ERS, and IRS. As a result they found that the gravity coefficient $J_{5}$ is responsible for the larger decrease in the variations of the frozen elements, while the atmospheric drag can induce perturbations in a long period of time. This analysis can improve the precision for prediction of the frozen elements, eccentricity, and argument of perigee of artificial satellites.

The collision of the Iridium and Cosmos satellites in 2009 showed the necessity of improving the orbit prediction methods. In order to improve these methods, D. Ortigosa et al. developed a scalable second-order analytical orbit propagator software based on modern and classical perturbation methods. Firstly, they considered only the perturbations of the zonal harmonic coefficients derived from the Earth's gravity potential. This first test enables them to analyze the software and to determine its limits when classical problems, such as the critical inclination, are assumed. They used two different methods: the first removes the short-period terms due to the mean anomaly (the classical Delaunay normalization, DN) and the second one removes the shortperiod terms through the Krylov-Bogoliubov-Mitropolsky method (KB). Both methods were explored to analyze the errors produced by the propagation of the TLE catalogue. They concluded that the KB is more robust than $\mathrm{DN}$ in most of the tests over 7 days.

L. C. G. Souza and J. Fonseca studied how the dynamics and the control algorithm strategy of the reaction wheels with respective DC motor can influence the performance and robustness of the satellite attitude control system in three axes. In order to do this, a nonlinear 3-dimensional simulator model based on the state-dependent Riccati equation method is developed taking into account the reaction wheel parameters. By the analysis it is possible to conclude that the increasing the reaction wheel gain velocity feedback has better performance than the current feedback. It is important to say that the reaction wheel gain is a function of its axis since the inertia moments are different for each axis. Then the simulations results have shown that the reaction wheels with speed feedback compensation have improved the satellite attitude control system performance and robustness.

R. C. Domingos et al. used an approach related to orbital maneuvers in their paper. The problem is solved by considering the orbital evolution of a spacecraft perturbed by a third body in an elliptical orbit. The evolution of the spacecraft orbit is obtained by three different models: the restricted elliptical three-body problem and the singleaveraged and double-averaged models. Therefore, a double objective is reached by the present research: the measurement of the influence of the eccentricity of the perturbing body in the fuel consumption required by the station-keeping maneuvers and the validation of the averaged methods when applied to the problem of predicting orbital maneuvers. Impulsive MANEUVERS, with one or two impulses, and low thrust maneuvers, with one or two propulsion arcs, were used. The results showed that the averaged models are good to make predictions for the orbital maneuvers when high inclination orbits are considered, but they lose some quality when near equatorial and near critical inclination orbits are used. The eccentricity of the third body plays an important role, by increasing the effects of the perturbation and the fuel consumptions required for the station-keeping maneuvers. It is also shown that the use of more frequent maneuvers decreases the annual cost of the station in order to keep the correct eccentricity. The present theory is indicated to study the dwarf planet, Haumea, because it has a moon with large eccentricity.

M. N. Pontuschka and I. M. da Fonseca studied a FDIR for an IMU (inertial measurement unity) having in mind space applications. The focus is on the gyro signal analysis and the validation of the filtering algorithms. A good agreement was obtained in the results. This problem is important for both manned and unmanned space missions, because the implementation of a FDIR is necessary to make sure that a dependable and autonomous system with minimal risks of failures is available. To perform this task, each component has a set of criteria for fault detection and isolation. After that the actions are defined and implemented with the goal of recovering the fault.

I. Yang et al. proposed a robust nonlinear dynamic inversion control technique to maintain the relative position of spacecraft during a formation flying. The method is based on nonlinear dynamic inversion, which is a nonlinear control method that substitutes the original dynamics by a dynamics selected by the user. Since nonlinear dynamic inversion removes nonlinearities in the model by inverting the original dynamics, it is not necessary to design controllers for each equilibrium point. The performance of the method proposed in the present paper is then evaluated by simulations made in spacecraft formation flying problems.

F. Venditti and A. F. B. A. Prado studied the orbital dynamics around irregular bodies. This is an important topic nowadays, because, in general, celestial bodies are not perfect spheres and, in particular, small celestial bodies (asteroids, comets, etc.) are highly nonspherical. To model those bodies is not an easy task, because it is hard to learn the exact shape based on observations made from Earth. An estimation of their shapes can be obtained as a sum of known geometric shapes. In particular, some three-dimensional figures have analytical equations for the potential, such as the cube, which is considered here. The results show mappings indicating the orbits that are more and less perturbed and so indicating 
the ones that have a good potential to minimize stationkeeping maneuvers. Those points are found and they can be used for constellations of nanosatellites.

G. Arantes Jr. and L. S. Martins-Filho studied the problem of noncooperative rendezvous and docking/berthing (RVD/B). The main goal is to design the guidance, navigation, and control for the chaser in this problem. It is considered out-of-plane maneuvers in distinct phases of the mission, including the chaser far approach, as well as the closer approach final berthing. Guidance solutions are searched for each phase based on the Closhessy-Willtshire (CW) equations. For the control, a linear quadratic regulator approach (LQR) is used. At the phase of final berthing, an attitude tracker is tested based on a proportional derivative (PD) form, where the goal is to synchronize the chaser and target attitudes. Finally, the paper analyzes the performance of both controllers.

J. P. S. Carvalho et al. studied problems related to space missions that visit Europa, which constitute an important topic nowadays, because several missions to study this body are currently under investigation. The paper searches for the less perturbed circular orbits around Europa, based on the integral of the perturbing forces over the time. The value of this integral depends on the force model used and on the specific orbit of the spacecraft. The disturbing forces included in the model are third body perturbation from Jupiter and the $J_{2}, J_{3}$, and $C_{22}$ terms of the gravity field of Europa. Numerical studies are made and the results map the locations of the less perturbed orbits. Using the results, they found near-circular frozen orbits that present smaller amplitudes in the variations of the orbital elements.

A. G. de Souza and L. C. G. de Souza designed the attitude control system (ACS) for a spacecraft considering flexible solar panels, antennas, mechanical manipulators, and tanks with fuel. A very important interaction exists between the fuel slosh motion, the flexible motion of the panels, and the satellite rigid motion during orbital maneuvers. This is a fluid-structure interaction (FSI) problem, where some deformable or movable structure interacts with an internal fluid. The consequence of this interaction is that the design of the spacecraft controller needs to use the limits between these three different motions. The paper develops a mathematical model to describe the motion of a satellite in a fixed plane.

D. Wang et al. studied the interception problem using a "two-body dynamics" with an upper-bounded tangent impulse for the interceptor, assumed to be in an elliptic parking orbit, to send the chaser to collide with a noncooperative target travelling in a hyperbolic orbit. The first step is to obtain four special initial true anomalies where the velocity vectors are parallel to either of the asymptotes of the hyperbolic orbit of the target body. They are obtained using the Newton-Raphson method. The equation for the time of flight is solved using the secant method for a single-variable piecewise function. All the possible solutions are obtained considering different positions for the impulse, the sphere of influence of the Earth, and an upper bound for the fuel consumption.

$\mathrm{X}$. Yue et al. proposed a numerical method to find periodic orbits for the satellite relative motion using the time domain collocation (TDC) method to find the periodic solutions of an exact nonlinear relative model. The method starts with the initial conditions for the periodic relative orbits obtained by the Clohessy-Wiltshire (C-W) equations or the Schauner-Hemple (T-H) equations. Then, they are refined with the proposed approach, to generate almost bounded orbits. After obtaining these orbits, a method that is based on the least-squares concept is proposed to obtain projected closed orbits (PCO). Those orbits are a reference for the relative motion control. Performing several numerical simulations, it is shown that the presented TDC method of searching periodic orbits is simple and effective.

J. C. Sampaio et al. studied spacecraft protection and collision avoidance, which is a very important problem nowadays. The mathematical model includes several disturbances and resonances that affect the motion of these objects. They are assumed to be distributed in several altitudes. In particular, the objects that are located in resonant orbital motions are studied. The resonant angles and periods associated with real motions are detailed, using the two-line elements of the NORAD. It gives more accurate information to build an analytical model that can better describe a specific resonance. The time evolution of the orbital elements, semimajor axis, eccentricity, and inclination are presented for a set of debris. It is possible to detect irregular motions through frequency analysis and also looking at the existence of different resonant angles.

\section{Acknowledgments}

The guest editors would like to thank the authors and reviewers who worked on the publication of the present special issue.

Antonio F. Bertachini A. Prado
Josep J. Masdemont
Maria Cecilia Zanardi
Silvia Maria Giuliatti Winter
Tadashi Yokoyama
Vivian Martins Gomes




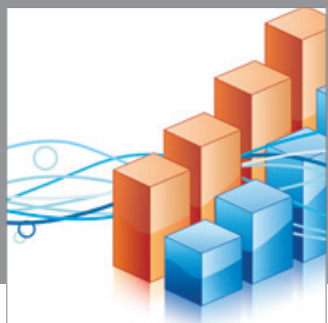

Advances in

Operations Research

mansans

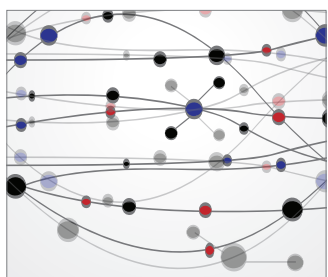

The Scientific World Journal
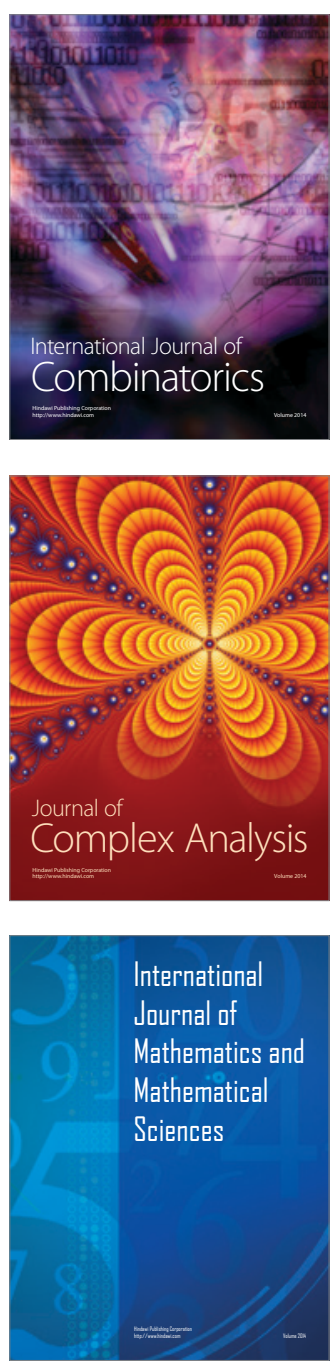
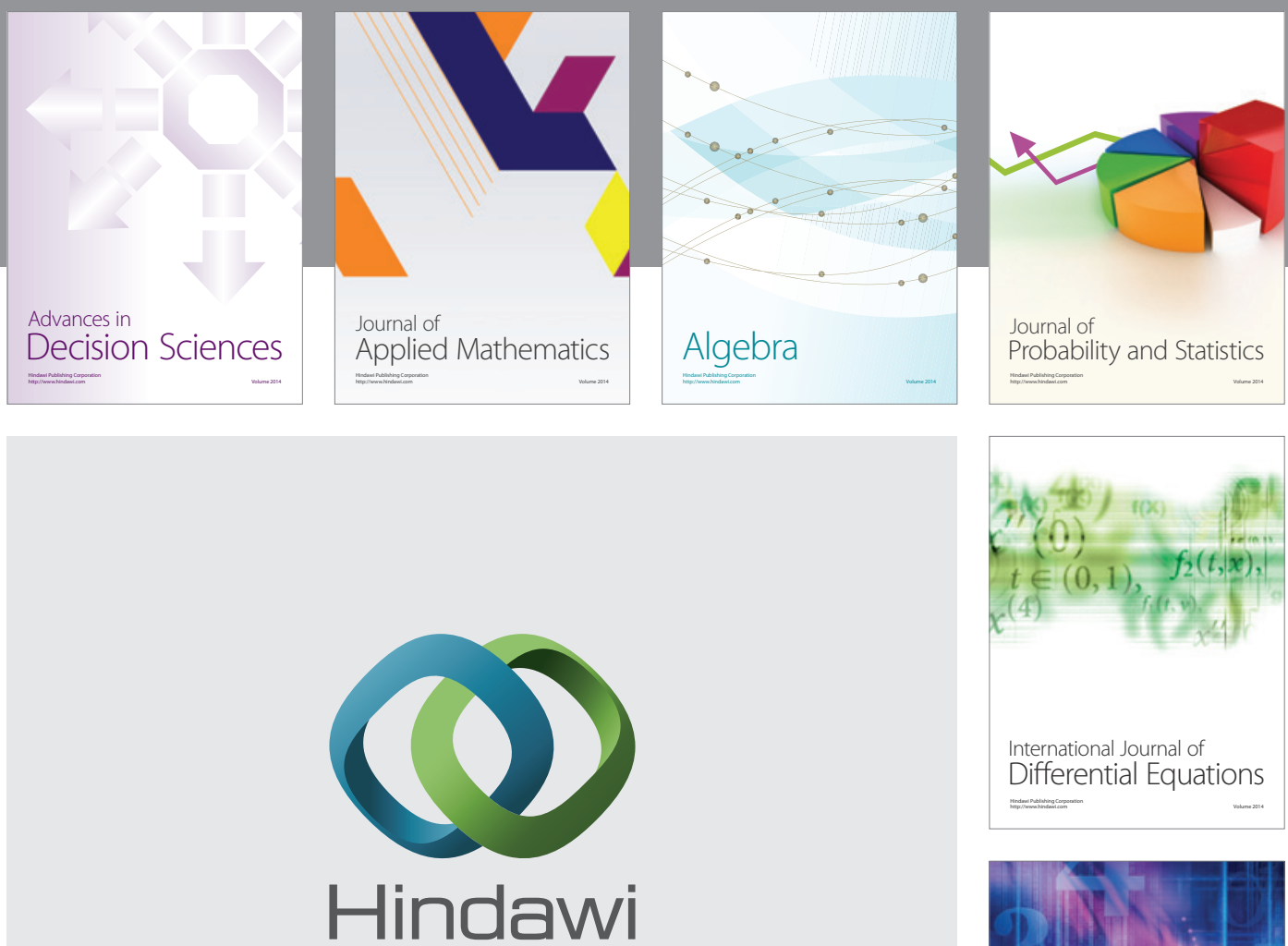

Submit your manuscripts at http://www.hindawi.com
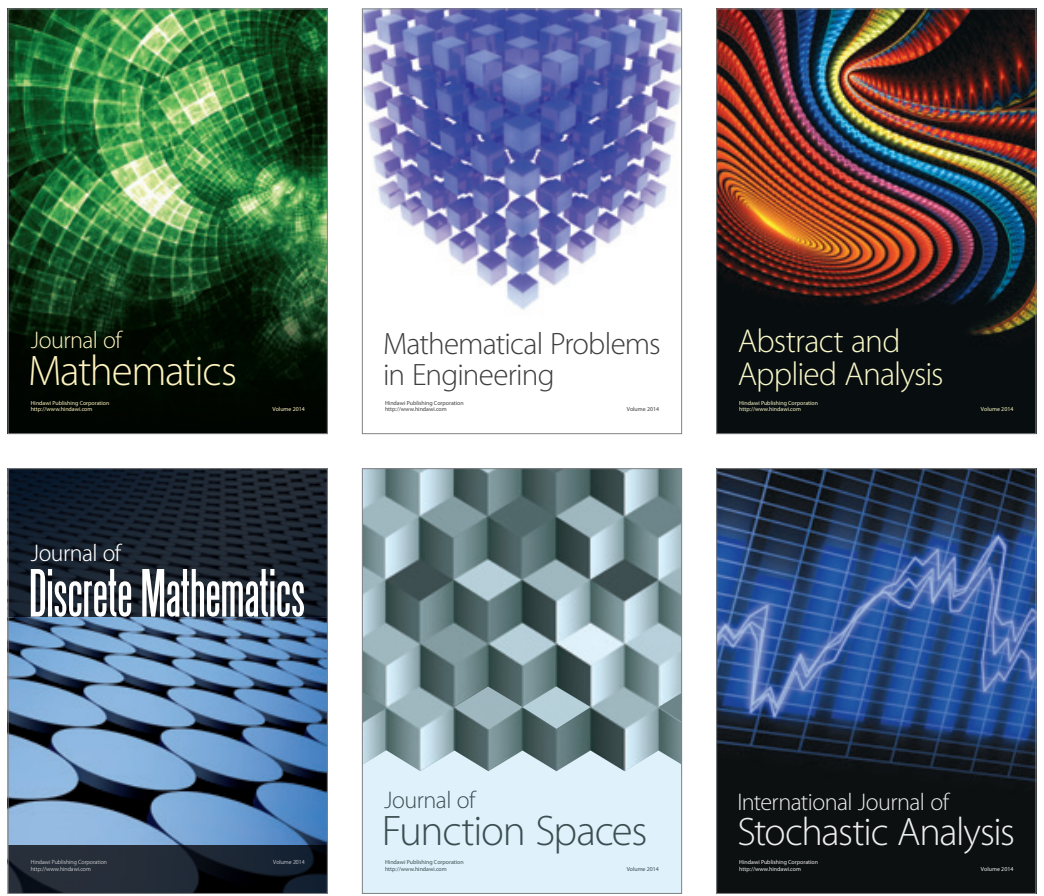

Journal of

Function Spaces

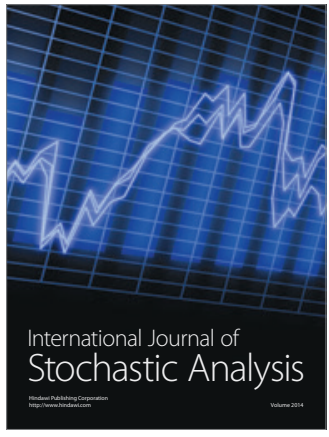

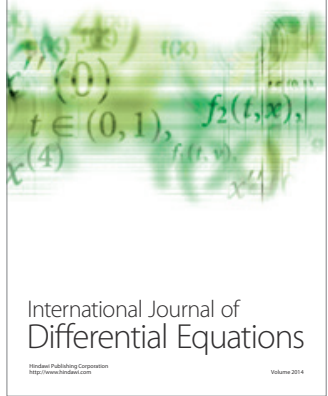
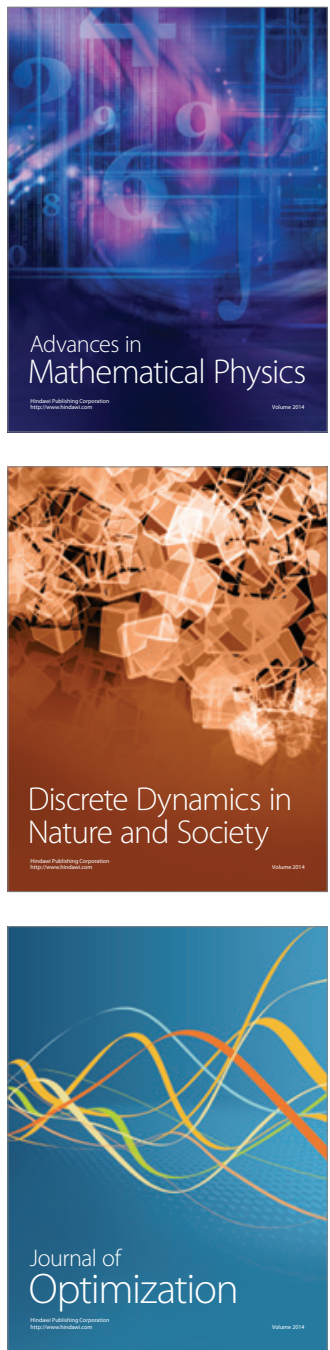